L. T. Hoa

Nagoya Math. J.

Vol. 110 (1988), 113-128

\title{
ON SEGRE PRODUCTS OF AFFINE SEMIGROUP RINGS
}

\author{
LÊ TUÂN HOA
}

\section{§. Introduction}

Let $N$ denote the set of non-negative integers. An affine semigroup is a finitely generated submonoid $S$ of the additive monoid $N^{m}$ for some positive integer $m$. Let $k[S]$ denote the semigroup ring of $S$ over a field $k$. Then one can identify $k[S]$ with the subring of a polynomial ring $k\left[t_{1}, \cdots, t_{m}\right]$ generated by the monomials $t^{x}=t_{1}^{x_{1}} \cdots t_{m}^{x_{m}}, x=\left(x_{1}, \cdots, x_{m}\right) \in S$.

Let $Q$ denote the field of rational numbers. Let $\sigma: Q^{m} \rightarrow Q$ be a linear functional such that $\sigma(S) \subseteq N$ and $\sigma(x)=0, x \in S$, implies $x=0$. Then one can define an $N$-grading on $k[S]$ by setting deg $t^{x}=\sigma(x)$ for all $x \in S$. Such a procedure is called specializing to an $N$-grading [13, p. 190].

If $T \subseteq N^{n}$ is another affine semigroup and $k[T]$ is specialized to an $N$-grading by a linear functional $\tau: Q^{n} \rightarrow Q$, then one can define a new affine semigroup $W \subseteq N^{m} \times N^{n}$ by setting

$$
W:=(S \times T) \cap F,
$$

where $F$ denotes the set of all elements $(x, y) \in Q^{m} \times Q^{n}$ with $\sigma(x)=\tau(y)$. We call $k[W]$ the Segre product of the $N$-graded rings $k[S]$ and $k[T]$ with respect to $\sigma$ and $\tau$ (cf. [9, p. 125]). The class of rings of the form $k[W]$ includes, for example, the usual Segre product of polynomial rings, the Segre-Veronese graded algebra and the Rees algebras of certain rings generated by monomials. Several authors have been dealt with the Cohen-Macaulayness and the Gorensteiness of Segre products of special classes of affine semigroup rings [1], [2], [3], [4], [16].

The main result of this paper is a combinatorial criterion for $k[W]$ to be a Cohen-Macaulay (res. Gorenstein) in terms of $S$ and $T$ (Theorem 2.1). It is based on a combinatorial criterion of [16] for an affine semigroup ring to be Cohen-Macaulay (res. Gorenstein) which uses certain simplicial complexes associated with the affine semigroup (see Section 1). We shall see that the associated simplicial complexes of $W$ are the joins

Received August 26, 1986. 
of the associated simplicial complexes of $S$ and $T$. This fact gives a topological meaning to the Segre product of affine semigroups and will play an essential role in the proof of the main result of this paper. If one of the rings $k[S]$ and $k[T]$ is Cohen-Macaulay and $\sigma(S)=\tau(T)=N$, the conditions of our criterion turn out to be rather simple (Theorem 3.1). From these conditions one can easily derive the results of [1], [2], [4], [16] on the Cohen-Macaulayness and Gorensteiness of Segre products of certain affine semigroup rings. Moreover, as a by-product of our investigation, we can also show that the Buchsbaumness of affine semigroup rings is dependent upon the characteristic of the basic field (Proposition 4.1). This is of some interest because only polynomial rings modulo ideals generated by square-free monomials were known to possess the same property [11]. (Cf. [10] and [16] for the Cohen-Macaulay case).

\section{§1. Preliminaries}

In this section, we recall some basic facts on affine semigroup rings.

Let $Z$ denote the set of integers. Let $G(S)$ denote the additive group in $Z^{m}$ generated by $S$ and put $r=\operatorname{rank}_{Z} G(S)$. In this paper, we always assume that $r \geq 2$.

If $A$ and $B$ are subsets of $G(S), A \pm B$ denotes the set of all elements of the forms $e \pm f$ with $e \in A, f \in B$, respectively. Consider the elements of $S$ as points in the space $Q^{m}$. Let $\mathscr{C}_{S}$ denote the convex rational polyhedral cone spanned by $S$ in $Q^{m}$. Then $\mathscr{C}_{S}$ is $r$-dimensional. Suppose that $P_{1}, \cdots, P_{p}$ are the facets of $\mathscr{C}_{s}$, i.e. the $(r-1)$-dimensional faces of $\mathscr{C}_{S}$. Set

$$
\begin{aligned}
& S_{i}=S-S \cap P_{i}, \\
& S^{\prime}=\bigcap_{i=1}^{p} S_{i} .
\end{aligned}
$$

Further, let $[1, p]$ denote the set of the integers $1, \cdots, p$. For every subset $J$ of $[1, p]$, set

$$
G_{J}^{S}=\bigcap_{i \in J} S_{i} \backslash \bigcup_{i \in J} S_{i},
$$

and let $\pi_{J}^{S}$ denote the simplicial complex of non-empty subsets $I$ of $J$ with the property $\bigcap_{i \in I} S \cap P_{i} \neq(0)$. Note that $\pi_{J}^{S}$ is called acyclic if the reduced homology group $\tilde{H}_{q}\left(\pi_{J}^{S} ; k\right)$ vanishes for all $q \geq 0$.

There is the following criterion for an affine semigroup ring to be 
Cohen-Macaulay.

Lemma 1.1 [16, Main Theorem]. Let $S$ be an arbitrary affine semigroup. Then $k[S]$ is a Cohen-Macaulay (res. Gorenstein) ring iff the following conditions are satisfied:

(i) $S^{\prime}=S$ (res. there exists an element $x \in G(S)$ such that $G_{[1, p]}^{S}=x$ $-S)$

(ii) For every non-empty proper subset $J$ of $[1, p], G_{J}^{S}=\varnothing$ or $\pi_{J}^{S}$ is acyclic.

If $S=S^{\prime}$, there is even a description of the local cohomology modules of $k[S]$ in terms of $G_{J}^{S}$ and $\pi_{J}^{S}$. To formulate it we need some more notations:

Given two $Z^{m}$-graded modules $M_{1}$ and $M_{2}$ over $k[S]$, one can define the $Z^{m}$-graded Segre product

$$
M_{1} \otimes M_{2}:=\bigoplus_{x \in Z^{m}}\left[M_{1}\right]_{x} \otimes_{k}\left[M_{2}\right]_{x},
$$

where $\left[M_{1}\right]_{x}$ and $\left[M_{2}\right]_{x}$ denote the $x$-graded piece of $M_{1}$ and $M_{2}$. Obviously, $M_{1} \otimes M_{2}$ can be considered as a $Z^{m}$-graded module over $k[S]=k[S] \otimes k[S]$. Note that if $A, B$ are arbitrary subsets of $G(S)$ such that $A+S \subseteq A$, $B+S \subseteq B$, then $k[A]$ and $k[B]$ can be considered as $Z^{m}$-graded modules over $k[S]$ and

$$
k[A] \otimes k[B]=k[A \cap B] .
$$

Let $D_{S, i}^{\cdot}$ denote the complex

$$
0 \longrightarrow D_{S, i}^{0} \stackrel{d}{\longrightarrow} D_{S, i}^{1} \longrightarrow 0
$$

where $D_{S, i}^{0}:=k[G(S)], D_{S, 1}^{1}:=k\left[G(S) \backslash S_{i}\right]$, and $d$ is the canonical map from $k[G(S)]$ to $k\left[G(S) \backslash S_{i}\right]=k[G(S)] / k\left[S_{i}\right], i=1, \cdots, p$. Put

$$
D_{S}^{\cdot}:=D_{S, 1}^{\cdot} \otimes \cdots \otimes D_{S, p}^{\cdot},
$$

which consists of the terms

$$
D_{S}^{j}=\underset{\substack{I \subseteq 1, p] \\ \# I=j}}{\bigoplus} D_{S, I}=\underset{\substack{I \subseteq[1, p] \\ \sharp I=j}}{\bigoplus} k\left[G(S) \backslash \bigcup_{i \in I} S_{i}\right]
$$

For simplicity, set $\pi^{S}=\pi_{[1, p]}^{S}$. Let $D_{\pi s}^{\cdot}$ denote the subcomplex of $D_{S}^{\cdot}$ consisting of the terms

$$
D_{\pi s}^{j}:=\underset{\substack{I \in \pi_{S} \\ \# I=j}}{\oplus} D_{S I}
$$


$j \geq 0$. Then we have

Lemma 1.2 [16, Lemma 3.2]. Suppose that $S^{\prime}=S . \quad$ Put $\mathfrak{m}_{s}:=k[S \backslash(0)]$. Then

$$
H_{\mathrm{w}_{S}}^{j}(k[S])=H^{j}\left(D_{\pi s}^{\cdot s}\right)
$$

for all $j \geq 0$.

In particular, one can express the graded piece $\left[H_{m_{S}}^{j}(k[S])\right]_{x}$ in terms of some simplicial subcomplexes $\pi_{J}^{S}$ of $\pi^{S}$ as follows.

Lemma 1.3 [16, Theorem 3.3]. For every $x \in G(S)$, set

$$
J_{x}:=\left\{i \in[1, p] ; x \notin S_{i}\right\} .
$$

Suppose that $S^{\prime}=S$. Then

$$
\left[H_{\mathrm{m}_{S}}^{j}(k[S])\right]_{x}=\tilde{H}_{j-2}\left(\pi_{J_{x}}^{S} ; k\right)
$$

for all $j>0$.

Note that the set of all elements $x \in G(S)$ such that $J_{x}=J$ for some fixed subset $J \subseteq[1, p]$ is just the set $G_{J}^{S}$ (cf. [16, Corollary 3.7]).

\section{§ 2. Main result}

Using the notations of the preceding sections, the main result of this paper may be formulated as follows.

Theorem 2.1. Let $p$ and $q$ are the numbers of facets of $\mathscr{C}_{S}$ and $\mathscr{C}_{T}$, res.. Then $k[W]$ is a Cohen-Macaulay (res. Gorenstein) ring iff the following conditions are satisfied:

(i) $\sigma\left(S^{\prime} \backslash S\right) \cap \tau\left(T^{\prime}\right)=\varnothing$ and $\tau\left(T^{\prime} \backslash T\right) \cap \sigma\left(S^{\prime}\right)=\varnothing$ (res. there exist elements $x \in G_{[1, p]}^{S}$ and $y \in G_{[1, q]}^{T}$ with $\sigma(x)=\tau(y)$ such that

$$
\left.\sigma\left(G_{[1, p]}^{S} \backslash(x-S)\right) \cap \tau\left(G_{[1, q]}^{T}\right)=\varnothing, \quad \tau\left(G_{[1, q]}^{T} \backslash(y-T)\right) \cap \sigma\left(G_{[1, p]}^{S}\right)=\varnothing\right) .
$$

(ii) For every pair of subsets $I \subseteq[1, p], J \subseteq[1, q]$ such that $(I, J) \neq$ $(\varnothing, \varnothing),([1, p],[1, q])$ and

$$
\sigma\left(G_{J}^{S}\right) \cap \tau\left(G_{J}^{T}\right) \neq \varnothing
$$

either $\pi_{I}^{S}$ or $\pi_{J}^{T}$ is acylic.

To prove Theorem 2.1, we need some auxiliary considerations.

Let $P_{1}, \ldots, P_{p}$ and $Q_{1}, \cdots, Q_{q}$ denote the facets of $\mathscr{C}_{S}$ and $\mathscr{C}_{T}$, respectively. Since: 


$$
\mathscr{C}_{W}=\left(\mathscr{C}_{S} \times \mathscr{C}_{T}\right) \cap F,
$$

$\mathscr{G}_{W}$ has the following $p+q$ facets:

$$
E_{i}= \begin{cases}\left(P_{i} \times \mathscr{C}_{T}\right) \cap F, & i=1, \cdots, p, \\ \left(\mathscr{C}_{S} \times Q_{i-p}\right) \cap F, & i=p+1, \cdots, p+q .\end{cases}
$$

Lemma 2.2. Put $W_{i}=W-W \cap E_{i}, i=1, \cdots, p+q$. Then

$$
W_{i}= \begin{cases}\left(S_{i} \times G(T)\right) \cap F, & i=1, \cdots, p, \\ \left(G(S) \times T_{i-p}\right) \cap F, & i=p+1, \cdots, p+q .\end{cases}
$$

Proof. We only need to prove that

$$
W_{1}=\left(S_{1} \times G(T)\right) \cap F .
$$

The conclusion $\subseteq$ is obvious. Conversely, each element of $\left(S_{1} \times G(T)\right)$ $\cap F$ has the form $\left(s-s_{1}, t-t^{\prime}\right)$, with $s \in S, s_{1} \in S \cap P_{1}, t, t^{\prime} \in T$ and $\sigma\left(s-s_{1}\right)=\tau\left(t-t^{\prime}\right)$. We may assume that $s_{1} \neq 0, t^{\prime} \neq 0$, Then $u=\sigma\left(s_{1}\right)$ $\geqq 1$ and $v=\tau\left(t^{\prime}\right) \geq 1$. Hence

$$
\begin{aligned}
\left(s-s_{1}, t-t^{\prime}\right) & =\left(s+(v-1) s_{1}-v s_{1}, t+(u-1) t^{\prime}-u t^{\prime}\right) \\
& =\left(s+(v-1) s_{1}, t+(u-1) t^{\prime}\right)-\left(v s_{1}, u t^{\prime}\right) \in W_{1},
\end{aligned}
$$

as required.

Lemma 2.3. Let $K$ be an arbitrary subset of $[1, p+q]$. Set $I=K \cap$ $[1, p]$ and $J=\{i-p ; i \in K, i>p\}$. Then

(i) $G_{K}^{W}=\left(G_{I}^{S} \times G_{J}^{T}\right) \cap F$,

(ii) $\pi_{K}^{W}=\pi_{I}^{S} * \pi_{J}^{T}$ (the join of $\pi_{I}^{S}$ and $\pi_{J}^{T}$ (see [12])).

Proof. (i) Straightforward.

(ii) By the definition of $\pi_{K}^{W}, \pi_{I}^{S}$, and $\pi_{J}^{T}$, it suffices to show that

$$
\bigcap_{i \in K} W \cap E_{i} \neq(0) \quad \text { iff } \bigcap_{i \in I} S \cap P_{i} \neq(0)
$$

and $\bigcap_{j \in J} T \cap Q_{j} \neq(0)$. First note that

$$
\bigcap_{i \in K} W \cap E_{i}=\left(\left(\bigcap_{i \in I} S \cap P_{i}\right) \times\left(\bigcap_{i \in J} T \cap Q_{j}\right)\right) \cap F .
$$

Suppose that $\bigcap_{i \in K} W \cap E_{i}$ contains an element $(x, y) \neq 0, x \in \bigcap_{i \in I} S \cap P_{i}$ and $y \in \bigcap_{j \in J} T \cap Q_{j}$ with $\sigma(x)=\tau(y)$. Then $x \neq 0$ and $y \neq 0$ because $\sigma(x)$ $=\tau(y)=0$ iff $x=y=0$. Conversely, if $\bigcap_{i \in I} S \cap P_{i}$ and $\bigcap_{j \in J} T \cap Q_{j}$ contain elements $x \neq 0$ and $y \neq 0$, then $(\tau(y) x, \sigma(x) y)$ is a non-zero element of $\bigcap_{i \in K} W \cap E_{i}$. 
Proof of Theorem 2.1. Note that $S^{\prime}=G_{\phi}^{S}, T^{\prime}=G_{\phi}^{T}$. Then, by Lemma 2.3(i),

$$
W^{\prime}=\left(S^{\prime} \times T^{\prime}\right) \cap F, \quad G_{[1, p+q]}^{W}=\left(G_{[1, p]}^{S} \times G_{[1, p]}^{T}\right) \cap F .
$$

Hence it is easy to check that $W^{\prime}=W$ (res. $G_{[1, p+q]}^{W}=(x, y)-W$ for some element $(x, y) \in G(W)$ ) iff condition (i) of Theorem 2.1 is satisfied. Let $K$ be an arbitrary non-empty proper subset of $[1, p+q]$ and $I, J$ as in Lemma 2.3. Then, by Lemma 2.3(i), $G_{K}^{W}=\varnothing$ iff $\sigma\left(G_{I}^{S}\right) \cap \tau\left(G_{J}^{T}\right)=\varnothing$. Moreover, using Lemma 2.3(ii) we get

$$
\tilde{H}_{s}\left(\pi_{K}^{W} ; k\right)=\sum_{i+j=s-1} \tilde{H}_{i}\left(\pi_{I}^{S} ; k\right) \otimes_{k} \tilde{H}_{j}\left(\pi_{J}^{T} ; k\right)
$$

for all $s \geq 0$ by [5, p. 126]. Therefore, $\pi_{K}^{W}$ is acyclic iff $\pi_{I}^{S}$ or $\pi_{J}^{T}$ is acyclic. Now, we can conclude that condition (ii) of Lemma 1.1 formulated for $W$ is equivalent to condition (ii) of Theorem 2.1. Hence, the statement follows from Lemma 1.1.

Remark. The canonical module of $k[W]$ can be expressed in terms of the ones of $k[S]$ and $k[T]$ as follows. By Lemma 2.3 and [16, Corollary $3.8]$.

$$
H_{\mathrm{m}_{W}}^{d}(k[W])=k\left[\left(G_{[1, p]}^{S} \times G_{[1, q]}^{T}\right) \cap F=k\left[G_{[1, p]}^{S}\right] \otimes k\left[G_{[1, q]}^{T}\right],\right.
$$

where $d=\operatorname{dim} k[W], \mathfrak{m}_{W}=k[W \backslash(0)]$. Hence

$$
K_{k[W]}=K_{k[S]} \otimes K_{k[T]},
$$

where $K_{k[W]}, K_{k[S]}$, and $K_{k[T]}$ denote the canonical $Z$-graded modules of $k[W], k[S]$, and $k[T]$, respectively.

The following example show that $k[S]$ and $k[T]$ needn't to be CohenMacaulay and even Buchsbaum rings if their Segre product with respect to some $Z$-gradings is a Cohen-Macaulay ring.

ExAmple 2.4. Let $S \subseteq N^{2}$ be generated by four elements $(5,0),(4,1)$, $(1,4)$, and $(0,5)$. Then $k[S]=k\left[t_{1}^{5}, t_{1}^{4} t_{2}, t_{1} t_{2}^{4}, t_{2}^{5}\right]$ is the homogeneous coordinate ring of a double projection of a Veronese variety. It is easy to see that $k[S]$ is not Cohen-Macaulay. By [15], $k[S]$ is even not Buchsbaum. Let $k[S]$ be specialized to and $N$-grading by the linear functional $\sigma: Q^{2}$ $\rightarrow Q, \sigma\left(x_{1}, x_{2}\right)=\left(x_{1}+x_{2}\right) / 5$. Then we have

$$
\begin{aligned}
& \sigma\left(S^{\prime} \backslash S\right)=\{1,2\}, \quad \sigma\left(G_{\phi}^{S}\right)=\sigma\left(S^{\prime}\right)=N, \\
& \sigma\left(G_{[1,2]}^{S}\right)=\{s \in Z ; s \leq-1\} .
\end{aligned}
$$


Let $T=N^{2}$ and let $k[T]=k\left[t_{1}, t_{2}\right]$ be specialized to an $N$-grading by the linear functional $\tau: Q^{2} \rightarrow Q, \tau\left(x_{1}, x_{2}\right)=3\left(x_{1}+x_{2}\right)$. Then $T^{\prime}=T$ and

$$
\begin{aligned}
& \sigma\left(T^{\prime}\right)=\tau\left(G_{\dot{\phi}}^{T}\right)=\{0,3,6, \cdots\}, \\
& \tau\left(G_{[1,2]}^{T}\right)=\{-3,-6, \cdots\} .
\end{aligned}
$$

Hence, by Theorem 2.1, the Segre product of $k[S]$ and $k[T]$ is a CohenMacauly ring.

Now it is natural to ask about properties of $N$-graded affine semigroup rings whose Segre products are Cohen-Macaulay rings.

To give a partial answer to this question, we shall need the notation of generalized Cohen-Macaulay rings (see [6]). Let $(A, \mathfrak{m})$ be a Noetherial local ring. Then $A$ is called a generalized Cohen-Macaulay ring if $l\left(H_{\mathrm{m}}^{i}(A)\right)<\infty$ for $i=0, \cdots, \operatorname{dim} A-1$. This notation is a generalization of that of Buchsbaum rings and many interesting properties were known above them. An affine semigroup ring $k[S]$ is called a generalized CohenMacaulay ring if the localization of $k[S]$ at the maximal ideal $\mathfrak{m}_{S}=$ $k[S \backslash(0)]$ is a generalized Cohen-Macaulay ring.

Corollary 2.5. Suppose that $\sigma(S)=\tau(T)=N$. If $k[W]$ is a CohenMacaulay ring, $k[S]$ and $k[T]$ are generalized Cohen-Macaulay rings.

Proof. By the definition of $S^{\prime}$ and $T^{\prime}$ one knows that every element of $S^{\prime}$ res. $T^{\prime}$ lies in the rational convex cone $\mathscr{C}_{S}$ res. $\mathscr{C}_{T}$. Hence $\sigma\left(S^{\prime}\right)$ $=N$ res. $\tau\left(T^{\prime}\right)=N$ if $\sigma(S)=N$ res. $\tau(T)=N$. From this and by condition (i) of Theorem 2.1 one easily gets $S=S^{\prime}$ and $T=T^{\prime}$. Hence, by Lemma 2.3, $H_{\mathrm{m}_{S}}^{i}(k[S])$ is concentrated in degrees $\sigma(x), x \in G_{I}^{S}$ for some $I \subseteq$ $[1, p]$ with $\tilde{H}_{i-2}\left(\pi_{I}^{S} ; k\right) \neq 0, i<\operatorname{dim} k[S]$. Since $\pi_{I}^{S}$ and $\pi^{T}$ are not acyclic [16, Corollary 3.6], from condition (ii) of Theorem 2.1 we get

$$
\sigma\left(G_{I}^{S}\right) \cap \tau\left(G_{[1, q]}^{T}\right)=\varnothing
$$

Note that if $y$ is an arbitrary element of $G_{[1, q]}^{T}=G(T) \backslash \bigcup_{i=1}^{q} T_{i}$, then $y-z$ also belongs to $G_{[1, q]}^{T}$ for all $z \in T$. Since $\tau(T)=N$, we can find an integer $s$ such that $\tau\left(G_{[1, q]}^{T}\right)$ contains the set of integers $\leq s$. Hence $\sigma\left(G_{I}^{S}\right)$ contains only integers $\geq s$. By [17, Lemma 2.2] we can conclude that $\exists_{\mathrm{m}_{S}}^{i}(k[S])$ is finitely generated. Hence $k[S]$ is a generalized Cohen-Macaulay ring. Similarly, one can also show that $k[T]$ is a generalized CohenMacaulay ring. 


\section{§3. The case one ring being Cohen-Macaulay}

In this section we will consider the case $k[T]$ being a Cohen-Macaulay ring with $\tau(T)=N$. We shall see that the conditions of Theorem 2.1 can be simplified by means of the following invariants of $S$ and $T$ :

$$
\begin{aligned}
& a(S):=\max \sigma\left(G_{[1, p]}^{S}\right), \\
& a(T):=\max \tau\left(G_{[1, q]}^{T}\right) .
\end{aligned}
$$

Theorem 3.1. Suppose that $k[T]$ is a Cohen-Macaulay (res. Gorenstein) ring with $\tau(T)=N\left(\right.$ res. $\sigma(S)=\tau(T)=N$ or $\tau\left(T \cap Q_{i}\right)=N$ for all $\left.i \in[1, q]\right)$. Then $k[W]$ is a Cohen-Macaulay (res. Gorenstein) ring iff the following conditions are satisfied:

(i) $S^{\prime}=S$ (res. $G_{[1, p]}^{S}=x-S$ for some element $x \in G(S)$ such that $\sigma(x)=a(T))$,

(ii) $a(S)<0$,

(iii) $a(T)<0$,

(iv) For every non-empty proper subset $I$ of $[1, p], \sigma\left(G_{I}^{S}\right) \subseteq[a(T)+1$, - 1] or $\pi_{I}^{S}$ is acyclic.

We shall need the following consequences of the condition $\tau(T)=N$.

Lemma 3.2. Suppose that $\tau(T)=N$. Then

(i ) $\sigma\left(S^{\prime} \backslash S\right) \cap \tau\left(T^{\prime}\right)=\varnothing$ iff $S^{\prime}=S$.

(ii) $\tau\left(G_{[1, q]}^{T}\right)=\{s \in Z ; s \leq a(T)\}$.

(iii) For an element $x \in G_{[1, q]}^{S}$ with $\sigma(x) \leq a(T)$,

$$
\sigma\left(G_{[1, p]}^{S} \backslash(x-S)\right) \cap \tau\left(G_{\lceil 1 . o 7}^{T}\right)=\varnothing
$$

iff $G_{[1, p]}^{S}=x-S$.

Proof. To (i). See the proof of Corollary 2.5.

To (ii). Let $x \in G_{[1, q]}^{T}$ such that $\tau(x)=a(T)$. Since

$$
G_{[1, q]}^{T}=G(T) \backslash \bigcup_{i=1}^{q}\left(T-T \cap Q_{i}\right),
$$

$x-y \in G_{[1, q]}^{T}$ for all $y \in T$. Since $\tau(y)$ can be any non-negative integer, we see that $\tau\left(G_{[1, q]}^{T}\right)=\{s \in Z ; s \leq a(T)\}$.

To (iii). We only need to prove the implication $\Rightarrow$. Since $x \in G_{[1, p]}^{S}$, $x-S \subseteq G_{[1, p]}^{S}$ as shown above. It remains to show that $G_{[1, p]}^{S} \backslash(x-S)=\varnothing$. If $u \in G_{[1, p]}^{S} \backslash(x-S)$, then $\sigma(u)>a(T)$ because of (ii) and $\sigma\left(G_{[1, p]}^{S} \backslash(x-S)\right.$ ) $\cap \tau\left(G_{[1, q]}^{T}\right)=\varnothing$. For each $i=1, \cdots, p$, one can choose a non-zero element 
$s_{i}$ in $S \cap P_{i}$. Replacing $s_{i}$ by $n s_{i}$ for $n$ sufficienlty large, we may assume that $\sigma\left(s_{i}\right)>-a(T)+\sigma(u)$. Then $u-s_{i} \in G_{[1, p]}^{S}$ and $\sigma\left(u-s_{i}\right)<a(T)$ for all $i=1, \cdots, p$. From this and by (ii) it follows that $u-s_{i} \in x-S$ for all $i=1, \cdots, p$ because

Hence

$$
\sigma\left(G_{[1, p]}^{S} \backslash(x-S)\right) \cap \tau\left(G_{[1, q]}^{T}\right)=\varnothing .
$$

$$
x-u \in \bigcap_{i=1}^{p}\left(S-S \cap P_{i}\right)=S^{\prime} .
$$

Hence $\sigma(x-u)=\sigma(x)-\sigma(u) \geq 0$ because $\sigma\left(S^{\prime}\right) \subseteq N$. Since $\sigma(x) \leq a(T)$, $\sigma(u) \leq a(T)$, a contradiction.

Proof of Theorem 3.1. First we will prove that condition (i) is equivalent to condition (i) of Theorem 2.1. By Lemma 1.1, $T^{\prime}=T$ res. $G_{[1, q]}^{T}$ $=y^{*}-T$ for some $y^{*} \in G_{[1, q]}^{T}$ (note that $\tau\left(y^{*}\right)=a(T)$ ). Hence the necessary part of the statement is trivial. For the proof of the sufficient part of the statement, we note that the Cohen-Macaulay case follows from Lemma 3.2(i). Concerning the Gorenstein case, let $x \in G_{[1, p]}^{S}$ and $y \in G_{[1, q]}^{T}$ such that $\sigma(x)=\tau(y)$,

and

$$
\sigma\left(G_{[1, p]}^{S} \backslash(x-S)\right) \cap \tau\left(G_{[1, q]}^{T}\right)=\varnothing,
$$

$$
\tau\left(G_{[1, q]}^{T} \backslash(y-T)\right) \cap \sigma\left(G_{[1, p]}^{S}\right)=\varnothing .
$$

Since $\sigma(x)=\tau(y) \leq a(T)$, by Lemma 3.2(iii) we have $G_{[1, p]}^{S}=x-S$.

If $\sigma(S)=N$, then again by Lemma 3.2(iii), $G_{[1, q]}^{T}=y-T$. From this it follows that $\tau(y)=a(T)$. Since $\sigma(x)=\tau(y), \sigma(x)=a(T)$.

If $\tau\left(T \cap Q_{i}\right)=N$ for all $i=1, \cdots, q$, we also have $\sigma(x)=\tau(y)=a(T)$. Indeed, by Lemma 1.1, $G_{[1, q]}^{T}=y^{*}-T$ for some $y^{*} \in G(T)\left(\tau\left(y^{*}\right)=a(T)\right)$. We shall show that $y=y^{*}$. Write $y=y^{*}-t$ for some $t \in T$, and assume, without restriction, that $T$ is a standard affine semigroup, i.e. $T \cap Q_{i}=$ $\left\{x \in T ; x_{i}=0\right\}$ for $i=1, \cdots, q[16$, Section 1]. If $t \neq 0$, there is an index $i \in[1, q]$ such that $t_{i}>0$. Then $T \cap Q_{i} \cap(t+T)=\varnothing$. Hence $\left(y^{*}-T\right.$ $\left.\cap Q_{i}\right) \cap(y-T)=\varnothing$. That implies

$$
y^{*}-T \cap Q_{i} \subseteq\left(y^{*}-T\right) \backslash(y-T)=G_{[1, q]}^{T} \backslash(y-T) .
$$

Note that $\tau\left(y^{*}\right)=a(T)$. Then

$$
\begin{aligned}
\tau\left(G_{[1, q]}^{T} \backslash(y-T)\right) & \supseteq \tau\left(y^{*}-T \cap Q_{i}\right)=a(T)-\tau\left(T \cap Q_{i}\right) \\
& =\{m \in Z ; m \leq a(T)\} .
\end{aligned}
$$


On the other hand, since $G_{[1, p]}^{S}=x-S, \sigma\left(G_{[1, p]}^{S}\right)$ contains sufficiently small negative integers. Therefore,

$$
\sigma\left(G_{[1, p]}^{S}\right) \cap \tau\left(G_{[1, q]}^{T} \backslash(y-T)\right) \neq \varnothing,
$$

a contradiction. So we must have $y=y^{*}$, as required. Thus, we have proved that (i) is equivalent to condition (i) of Theorem 2.1.

Further, by Lemma 1.1, for every non-empty proper subset $J$ of $[1, q], G_{J}^{T}=\varnothing$ or $\pi_{J}^{T}$ is acyclic. Hence it remains to check condition (ii) of Theorem 2.1 for every subset $K$ of $[1, p+q]$ such that $K \cap[p+1$, $p+q]=\varnothing$ or $K \supseteq[p+1, p+q]$. Put $I=K \cap[1, p]$. If $I=\varnothing$ or $[1, p]$. one has to check the conditions

$$
\begin{aligned}
& \sigma\left(G_{[1, p]}^{S}\right) \cap \tau\left(T^{\prime}\right)=\varnothing, \\
& \sigma\left(S^{\prime}\right) \cap \tau\left(G_{[1, q]}^{T}\right)=\varnothing .
\end{aligned}
$$

Since $\tau\left(T^{\prime}\right)=N$ and $\tau\left(G_{[1, q]}^{T}\right)=\{s \in Z ; s \leq a(T)\}$, these conditions are equivalent to (ii) and (iii), respectively. If $I$ is a non-empty proper subset of $[1, p]$, one has to check the condition

$$
\sigma\left(G_{I}^{S}\right) \cap \tau\left(T^{\prime} \cup G_{[1, q]}^{T}\right)=\varnothing,
$$

which is equivalent to (iv).

Remark. One can not delete the assumption $\sigma(S)=\tau(T)=N$ or $\tau\left(T \cap Q_{i}\right)=N$ for $i=1, \cdots, q$ in the Gorenstein case of Theorem 3.1. For example, let $S=T \subseteq N^{2}$ be the affine semigroups generated by $(4,0),(0,4)$, $(1,1)$ and let $k[S]$ (res. $k[T]$ ) be specialized to an $N$-grading by the linear functional $\sigma:\left(x_{1}, x_{2}\right) \mapsto x_{1}+x_{2}$ (res. $\left.\tau:\left(x_{1}, x_{2}\right) \mapsto\left(x_{1}+x_{2}\right) / 2\right)$. Then $\tau(T)=N$ and $\sigma(S)=\tau\left(T \cap Q_{1}\right)=\tau\left(T \cap Q_{2}\right)=2 N$. By Theorem 2.1, one can easily check that the Segre product $k[W]$ of $k[S]$ and $k[T]$ is a Gorenstein ring, although $G_{[1,2]}^{S}=(-1,-1)-S$ and $\sigma((-1,-1))=a(S)=-2<a(T)=$ -1 .

Corollary 3.3. Suppose that $k[S]$ and $k[T]$ are Cohen-Macaulay (res. Gorenstein) rings with $\sigma(S)=\tau(T)=N$. Then $k[W]$ is a Cohen-Macaulay (res. Gorenstein) ring iff $a(S)<0$ and $a(T)<0$ (res. $a(S)=a(T)<0$ ).

Proof. The proof immediately follows from Theorem 3.1 and Lemma 1.1 .

To illustrate the use of Corollary 3.3 we conisder the so-called Segre-Veronese graded algebras [2]. First, recall that the Veronese $k$ - 
algebra of type $(n, d)$ is the ring generated by all monomials of degree $d$ in $n$ variables over $k$. It is the semigroup ring of the affine semigroup

$$
S(n, d)=\left\{x \in N^{n} ; x_{1}+\cdots+x_{n} \equiv 0 \text { modulo } d\right\} .
$$

It is well known that $k[S(n, d)]$ is a Cohen-Macaulay ring. $\mathscr{C}_{S(n, d)}$ has $n$ facets and it is easy to see that

$$
G_{[1, n]}^{S(n, d)}=\left\{x \in Z^{n} ; x_{1}+\cdots+x_{n} \equiv 0 \text { modulo } d \text { and } x_{i}<0, i=1, \cdots, n\right\} .
$$

Hence, using Lemma 1.1, one can check that $k[S(n, d)]$ is Gorenstein iff $n \equiv 0$ modulo $d$ (see also [2], [8]). $k[S(n, d)]$ has a natural $N$-graded structure corresponding to the linear functional $x \mapsto\left(x_{1}+\cdots+x_{n}\right) / d$.

A Segre-Veronese graded algebra is the Segre product of Veronese algebras [2] with respect to this natural $N$-graded structure.

Corollary 3.4 ([1], [2]). The Segre product $k[W]$ of the Veronese algebras $k\left[S\left(n_{1}, d_{1}\right)\right], \cdots, k\left[S\left(n_{r}, d_{r}\right)\right]$ is a Cohen-Macaulay ring. It is Gorenstein iff

$$
n_{1} / d_{1}=\cdots=n_{r} / d_{r} \in N .
$$

Proof. The proof immediately follows from Corollary 3.3 and Lemma 2.3 (i) by induction on $r$.

Note that the statement that Segre products of polynomial rings are Cohen-Macaulay [4] is only a consequence of Corollary 3.4.

One can also use Theorem 3.1 to study the arithmetically CohenMacaulayness res. Gorensteiness of the blowing-up of a projective monomial variety. For every affine semigroup $S \subset N^{m}$ such that $k[S]$ can be specialized to an $N$-grading by a linear functional $\sigma$, one can introduce the following affine semigroup

$$
S_{\sigma}:=\left\{(x, i) \in N^{m+1} ; x \in S \text { and } \sigma(x) \geq i\right\} .
$$

$k\left[S_{\sigma}\right]$ is isomorphic to the graded algebra $\oplus_{i=0}^{\infty} I_{i}$ where $I_{i}$ denotes the ideal of $k[S]$ generated by elements of degree $\geq i$. Especially, if $S$ is generated by elements of degree one, then $k\left[S_{\sigma}\right]$ is the Rees algebra of $k[S]$.

It is not hard to see that $k\left[S_{\sigma}\right]$ is isomorphic to the Segre product of $k[S]$ and $k\left[t_{1}, t_{2}\right]=k\left[N^{2}\right]$ (with the natural $N$-graded structure). Hence one can apply Theorem 3.1 to give a criterion for $k\left[S_{\sigma}\right]$ to be Cohen-Macaulay (res. Gorenstein) in terms of $S$.

Corollary $3.5\left[16\right.$, Lemma 4.8]. Let $S_{\sigma}$ be as above. Then $k\left[S_{\sigma}\right]$ is a 
Cohen-Macaulay (res. Gorenstein) ring iff the following conditions are satisfied:

(i) $S^{\prime}=S$ (res. $G_{[1, p]}^{S}=x-S$ for some $x \in G(S)$ with $\sigma(x)=-2$ ),

(ii) $a(S)<0$,

(iii) For every non-empty proper subset $I$ of $[1, p], \sigma\left(G_{I}^{S}\right) \subseteq\{-1\}$ or $\pi_{I}^{S}$ is acyclic.

Proof. Put $T=N^{2}$ and let $\tau$ denote the linear functional $\left(x_{1}, x_{2}\right) \mapsto$ $x_{1}+x_{2}$. Then it is esay to see that

$$
\begin{gathered}
T \cap Q_{1}=\left\{\left(0, x_{2}\right) ; x_{2} \in N\right\}, \\
T \cap Q_{2}=\left\{\left(x_{1}, 0\right) ; x_{1} \in N\right\}, \\
G_{[1,2]}^{T}=\left\{\left(x_{1}, z_{2}\right) \in Z^{2} ; x_{1}<0 \text { and } x_{2}<0\right\}=(-1,-1)-T .
\end{gathered}
$$

Thus $k[T]$ is a Gorenstein ring with $a(T)=-2$ and $\tau(T)=\tau\left(T \cap Q_{1}\right)$ $=\tau\left(T \cap Q_{2}\right)=N$. Hence the statement follows from Theorem 3.1.

\section{§4. Buchsbaumness of affine semigroup rings}

In 1976 Reisner [10] obtained the surprising result that the CohenMacaulayness of polynomial rings modulo ideals generated by square-free monomials is dependent upon the characteristic of the ground field. Later, Solcan [11] showed the same phenomenon for the Buchsbaumness of such rings. By [16] we also know that the Cohen-Macaulayness of affine semigroup rings is dependent upon the characteristic of the ground field. However, one was unable to establish the same phenomenon for the Buchsbaumness of such rings. Now, it will be done by applying results of the preceding sections.

Recall that a local ring $A$ with maximal ideal m is called a Buchsbaum ring if for every system of parameters $x_{1}, \cdots, x_{d}(d=\operatorname{dim} A>0)$ of $A$

$$
\left(x_{1}, \cdots, x_{i-1}\right): x_{i}=\left(x_{1}, \cdots, x_{i-1}\right): \mathfrak{m},
$$

for $i=1, \cdots, d$.

Here, we will need the following properties of Buchsbaum rings:

(i) Let $(A, \mathfrak{m})$ be a Buchsbaum ring of dimension $d>0$. Then $\mathrm{m} H_{\mathrm{m}}^{i}(A)=0$ for all $0 \leq i<d[14]$.

(ii) Let $k$ be a field, $A=\oplus_{n \geq 0}[A]_{n}$ a Noetherian $N$-graded ring with $A_{0}=k$ and $\mathfrak{m}=\oplus_{n>0}[A]_{n}$. Suppose that there is an integer $n$ such that for $0 \leq i<d=\operatorname{dim} A$ and for every $j \neq n$

$$
\left[H_{\mathrm{m}}^{i}(A)\right]_{j}=0 \text {. }
$$


Then $A_{\mathrm{m}}$ is Buchsbaum [7]. In this case, we will also say, for short, that $A$ is a Buchsbaum ring.

Proposition 4.1. There exists an affine semigroup ring such that it is non-Cohen-Macaulay Buchsbaum if char $(k) \neq 2$, but it is non-Buchsbaum if $\operatorname{char}(k)=2$.

For the proof, we consider the following two examples.

ExAmple 4.2. Let $\Delta$ be the simplicial complex associated with the minimal triangulation of the projective plane:

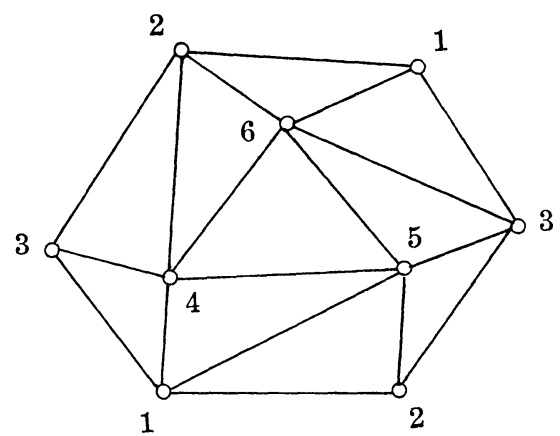

Then $\Delta$ can be considered as a subcomplex of the simplicial complex $\Delta_{[1,6]}$ of non-empty subsets of $[1,6] . \quad \Delta_{[1,6]} \backslash \Delta$ has the following 10 minimal simplexes: $\{1,2,3\},\{1,2,4\},\{1,3,5\},\{1,4,6\},\{1,5,6\},\{2,3,6\},\{2,4,5\}$, $\{2,5,6\},\{3,4,5\},\{3,4,6\}$. Consider the system of linear equations

$$
\left\{\begin{array}{l}
6\left(x_{1}+x_{2}+x_{3}\right)=x_{4}+x_{5}+x_{6}+15 x_{7} \\
6\left(x_{1}+x_{2}+x_{4}\right)=x_{3}+x_{5}+x_{6}+15 x_{8} \\
\cdots \ldots \ldots \ldots \ldots \ldots \ldots \ldots \ldots \ldots \\
6\left(x_{3}+x_{4}+x_{6}\right)=x_{1}+x_{2}+x_{5}+15 x_{16}
\end{array},\right.
$$

where the $i$-th equation corresponds to the $i$-th minimal simplex of $\Delta_{[1,6]} \backslash \Delta$ in the above order. Let $S$ be the affine semigroup of solutions $x \in N^{16}$ of this system of linear equations with $x_{i} \equiv x_{j}$ modulo 16 for all $i, j \in[1,16]$ and $x_{i} \neq 1,3$ for all $i \in[1,6]$.

Similary as in [16, Section 5], one sees that $\operatorname{dim} S=6, S^{\prime}=S, G_{[1,6]}^{S}$ $=\{(1, \cdots, 1),(3, \cdots, 3)\}, \pi_{[1,6]}^{S}=\Delta$ and that for every non-empty proper subset $J \neq[1,6]$ of $[1,16], G_{J}^{S}=\varnothing$ or $\pi_{J}^{S}$ is acyclic. Note that

$$
\tilde{H}_{q}(\Delta ; Z)= \begin{cases}0 & \text { if } q \neq 1, \\ Z_{2} & \text { if } q=1,\end{cases}
$$


and that

$$
H_{q}(\Delta ; k)=\left(H_{q}(\Delta ; Z) \otimes_{Z} k\right) \oplus \operatorname{Tor}_{Z}\left(H_{q-1}(\Delta ; Z) ; k\right)
$$

for all $q>0$ (the universal coefficient theorem [12]). Then, by Lemma 1.3,

$$
H_{\mathrm{m}_{S}}^{i}(k[S]=0
$$

for $i \neq 3,4,6$ and

$$
\left[H_{m_{S}}^{3}(k[S])\right]_{h}=\left[H_{m_{S}}^{4}(k[S])\right]_{h}=\left\{\begin{array}{cl}
Z_{2} \otimes_{z} k & \text { if } h=h_{1} \text { or } h_{3}, \\
0 & \text { if } h \neq h_{1}, h_{3},
\end{array}\right.
$$

where $h_{1}:=(1, \cdots, 1)$ and $h_{3}:=(3, \cdots, 3)$ are elements of $G(S)$. Thus, $k[S]$ is a Cohen-Macaulay ring if $\operatorname{char}(k) \neq 2$.

If $\operatorname{char}(k)=2$, let $h_{2}$ denote the element $(2, \cdots, 2)$ of $S$. Since

$$
\left[D_{S, I}\right]_{h_{1}}=\left[D_{S, I}\right]_{h_{3}}= \begin{cases}k & \text { if } I \subseteq[1,6], \\ 0 & \text { if } I \not[[1,6],\end{cases}
$$

the multiplication by $h_{2}$ induces an isomorphism of complexes:

$$
\left[D_{\pi}^{\cdot}\right]_{h_{1}} \stackrel{h_{2}}{\longrightarrow}\left[D_{\pi}^{\cdot}\right]_{h_{3}} .
$$

Since by Lemma 1.2 ,

$$
\left[H_{\mathrm{m}_{S}}^{i}(k[S])\right]_{x}=H^{i}\left(\left[D_{\pi}^{\cdot}\right]_{x}\right)
$$

for every element $x \in G(S)$, we get

$$
h_{2}\left[H_{\mathrm{m}_{S}}^{i}(k[S])_{h_{1}}=\left[H_{\mathrm{m}}^{i} S(k[S])\right]_{h_{3}} .\right.
$$

In particular, $h_{2} \cdot\left[H_{\mathrm{m}_{S}}^{i}(k[S])\right]_{h_{1}} \neq 0$. Therefore, $k[S]$ is a non-Buchsbaum ring.

ExAmple 4.3. Let $S$ be as in Example 4.2, and let $\sigma$ denote the linear functional

$$
\left(x_{1}, \cdots, x_{16}\right) \longmapsto\left(x_{1}+\cdots+x_{16}\right) / 16 .
$$

Then $\sigma\left(G_{\phi}^{S}\right)=\sigma\left(S^{\prime}\right)=N$ and $\sigma\left(G_{[1,6]}^{S}\right)=\{1,3\}$. Moreover, since

$$
\begin{aligned}
G_{[1,16]}^{S}= & G(S) \backslash \bigcup_{i=1}^{16} S_{i} \\
= & \left\{x \in G(S) ; x_{i}<0 \text { or } x_{i}=1,3 \text { for } i \in[1,6], x_{j}<0 \text { for } j \in[7,16]\right. \\
& \text { and } \left.x_{i} \equiv x_{j} \text { modulo } 16\right\},
\end{aligned}
$$

it is easy to see that $\sigma\left(G_{[1,16]}^{S}\right)=\{m \in Z ; m \leq-1\}$. 
Now let $T \subseteq N^{2}$ be the affine semigroup generated by $(3,0),(2,1)$, $(0,3)$. Let $k[T]$ be specialized to an $N$-grading by the linear functional $\tau:\left(x_{1}, x_{2}\right) \mapsto\left(x_{1}+x_{2}\right) / 3$. Then by Lemma 1.1 , it is easy to see that $k[T]$ is a Cohen-Macaulay ring and $\tau(T)=N$ and $\tau\left(G_{[1,2]}^{T}\right)=\{m \in Z ; m \leq 0\}$.

Let $k[W]$ denote the Segre product of $k[S]$ and $k[T]$ with respect to the functionals $\sigma$ and $\tau$. By Lemma 2.3, Example 4.2, and the above formulas, there are only two non-empty proper subsets $K$ of $[1,18]$ such that $G_{K}^{W} \neq \varnothing$ and $\pi_{K}^{W}$ is not acyclic. They are $[1,6]$ and $[17,18]$. We have

$$
\begin{aligned}
G_{[1,6]}^{W}= & \left\{\left(h_{1}, x_{1}\right),\left(h_{3}, x_{3}\right) ; x_{1}, x_{3} \in T, \tau\left(x_{1}\right)=1 \text { and } \tau\left(x_{3}\right)=3\right\}, \\
& G_{[17,18]}^{W}=\{(0, \cdots, 0,1,-1)\} .
\end{aligned}
$$

where $h_{1}, h_{3}$ are as in Example 4.2 .

Since $W=W^{\prime}$, by Lemma 1.3 and [16, Corollary 3.6] we ge,

$$
H_{\mathrm{m} W}^{i}(k[W])=0
$$

for $i \neq 2,3,4,7$ and

$$
\begin{gathered}
{\left[H_{\mathrm{m} w}^{2}(k[W])\right]_{w}= \begin{cases}k & \text { if } w=(0, \cdots, 0,1,-1), \\
0 & \text { if } w \neq(0, \cdots, 0,1,-1),\end{cases} } \\
{\left[H_{\mathrm{m} w}^{3}(k[W])\right]_{w}=\left[H_{\mathrm{m} w}^{4}(k[W])\right]_{w}=\left\{\begin{array}{cc}
Z_{2} \otimes_{Z} k & \text { if } w \in G_{[1,6]}^{W}, \\
0 & \text { if } w \notin G_{[1,6]}^{W}
\end{array}\right.}
\end{gathered}
$$

From this it follows that $k[W]$ is a non-Cohen-Macaulay Buchsbaum ring if char $(k) \neq 2$. If char $(k)=2$ one can see, similarly as in Example 4.2, that $k[W]$ is non-Buchsbaum.

Acknowledgement. I would like to thank Prof. Ngô Viêt Trung for his advices and encouragement. Thanks are also due to the referee for some constructive suggestions.

\section{REFERENCES}

[1] Badescu, L., On certain isolated singularities, Nagoya Math. J., 61 (1976), 205-220.

[2] Barcanescu, S., Gorensteiness of Segre-Veronese graded algebras, preprint 1979.

[ 3 ] Barshay, J., On the zeta function of biprojective complete intersections, Trans. Amer. Math. Soc., 135 (1969), 447-458.

[ 4 ] Chow, W. L., On unmixedness theorem, Amer. J. Math., 86 (1964), 799-822.

[ 5 ] Cooke, G. E. and Finney, R. L., Homology of cell complexes, Math. Notes, Princeton Univ. Press 1967.

[6] Cuong, N. T., Schenzel, P. and Trung, N. V., Verallgemeinerte Cohen-MacaulayModuln, Math. Nachr., 85 (1978), 57-73. 
[ 7 ] Goto, S., Buchsbaum rings of maximal embedding dimension, J. Algebra, 76 (1982), 494-508.

[ 8 ] — and Watanabe, K., On graded rings I, J. Math. Soc. Japan, 30 (1978), 179-213.

[ 9 ] Hartshorne, R., Algebraic geometry, Springer-Verlag, 1977.

[10] Reisner, G., Cohen-Macaulay quotients of polynomial rings, Adv. in Math., 21 (1976) , 30-49.

[11] Solcan, S., Über die Abhängigkeit der Buchsbaum-Eigenschaft von der Charakteristik des Grundkörpers, Math. Slovaka, 31 (1981), 437-444.

[12] Spanier, E. H., Algebraic topology, McGraw-Hill, 1966.

[13] Stanley, R. P., Linear diophantine equations and local cohomology, Invent. Math., 68 (1982), 175-193.

[14] Stückrad, J. and Vogel, W., Toward a theory of Buchsbaum singularities, Amer. J. Math., 100 (1978), 726-746.

[15] Trung, N. V., Classification of the double projections of Veronese varieties, J. Math. Kyoto Univ., 22 (1983), 567-581.

[16] Trung, N. V. and Hoa, L. T., Affine semigroups and Cohen-Macaulay rings generated by monomials, Trans. Amer. Math. Soc., 298 (1986), 145-167.

[17] Trung, N. V. and Ikeda, S., When is the Rees algebra Cohen-Macaulay, preprint.

Institute of Mathematics

P. O. Box 631, Bò Hô, Hanoi, Vietnam

Current address:

Sektion Mathematik

M. L. Universität Halle

Halle $(S) 4010$

$D D R$ 